\title{
O estado da arte das pesquisas em esportes coletivos para pessoas com deficiência: uma revisão sistemática
}

The state of art in researchers in disabled sports: a sistematic review

\author{
Mário Antônio de Moura Simim ${ }^{1,2,}$ \\ Bruno Victor Corrêa da Silva ${ }^{3}$ \\ Lucas Alves Facundo ${ }^{4}$ \\ Lidiane Aparecida Fernandes ${ }^{4}$ \\ Gustavo Ribeiro da Mota ${ }^{5}$
}

\section{Resumo}

Objetivo: Revisar a produção científica a respeito de modalidades esportivas coletivas para pessoas com deficiência e sugerir direcionamentos para estudos futuros. Métodos: Estudos foram identificados a partir de busca sistemática em três bases de dados. A pesquisa foi limitada para os idiomas inglês e português, e a estudos publicados até janeiro de 2018. Os trabalhos foram sumarizados e analisados em relação a frequência absoluta e relativa em 13 áreas de concentração. Resultados: Foram incluídos no estudo o total 348 trabalhos após exclusão dos estudos duplicados e que não atenderam os critérios de inclusão. Basquete, Rugby em cadeira de rodas, Futebol para Amputados e Goalball foram os esportes coletivos que apresentaram a maior frequência de estudos após síntese qualitativa. Análise de desempenho esportivo, aspectos fisiológicos e psicológicos foram as principais áreas de concentração dos estudos. Conclusão: Concluímos que a literatura é escassa na investigação das modalidades Futebol de cinco, Futebol de sete, Futebol em cadeira de rodas e Handebol em cadeira de rodas. Sugerimos que pesquisadores avancem nos conhecimentos fisiológicos, de desempenho esportivo e de psicologia do esporte em todas modalidades investigadas, mas que também desenvolvam estudos relativos a saúde e qualidade vida, nutrição esportiva, fisioterapia, tecnologia no esporte, teste e avaliações e nos aspectos sociais do esporte adaptado.

Palavras-Chave: paradesporto, paralímpico, atividade física adaptada.

\section{Abstract}

Objective: To review the scientific research regarding collective sports modalities for people with disabilities and to suggest directions for future studies. Methods: Studies were identified from a systematic search of three databases. The research was limited to the english and portugueselanguages, and published up to January 2018. The papers were summarized and analyzed in relation to absolute and relative frequency in 13 concentration areas. Results: A total of 348 studies were included in the study after exclusion from the duplicate studies and did not meet the inclusion criteria. Wheelchair basketball, wheelchair rugby, amputee soccer and goalball were the collective sports that presented the highest frequency of studies after qualitative synthesis. Sports performance analysis, physiological and psychological aspects were the main focus areas of the studies. Conclusion: We conclude that the literature is scarce in the investigation of sports Football 5-a-side, Football 7-a-side, power soccer and in wheelchair handball. We suggest that researchers advance their sports performance analysis, physiological, and sports psychology knowledge in all investigated sports, but also development studies related to health and quality of life, sports nutrition, physiotherapy, technology in sports, test and evaluations, and social aspects of sport adapted.

Key-words: para-sport, paralympic, adapted physical activity.

\section{*Autor correspondente}

Instituto de Educação Física e Esportes, Universidade Federal do Ceará, Av. Mister Hull s/n, Parque Esportivo, Bloco 320, Campus do Pici. CEP.: 60455-760. Fortaleza, Ceará, Brasil.

e-mail: mario.simim@ufc.br

Conflito de interesses

Os autores declararam não haver conflito de interesses.

\section{Processo de arbitragem}




\section{Introdução}

O esporte praticado por pessoas com deficiência (PCDs) teve seu início após a Segunda Guerra Mundial, embora já se promovessem atividades esportivas para PCDs antes da década de 40 ${ }^{1}$. O Esporte Adaptado ou Paradesporto é compreendido como prática que oportuniza às PCDs o alcance de novos horizontes e perspectivas de vida por meio de vivências motoras, psicológicas e sociais diversificadas ${ }^{2,3}$. A terminologia "Esporte Adaptado" é utilizado apenas no Brasil e consiste na possibilidade de prática esportiva para PCDs com modificações relacionadas às regras da modalidade ou a maneira como a modalidade se desenvolve $e^{1,4}$. Adicionalmente, o esporte para PCDs é geralmente dividido por grupos de deficiência específicos nos quais cada grupo tem história distinta, organização, programa de competição e abordagem ao esporte diferentes. Registra-se atualmente movimentos esportivos específicos para pessoas surdas, para deficientes físicos, deficientes visuais e para pessoas com deficiência intelectual $^{5}$.

Uma grande variedade de esportes foi adaptada para ser praticada por pessoas com várias deficiências, assim como outras modalidades foram criadas exclusivamente para PCDs ${ }^{5}$. Contudo, em cada movimento diferentes esportes são praticados em diferentes níveis; por exemplo, nem todos os esportes do movimento paralímpico fazem parte dos Jogos Paralímpicos. Além disso, muitos esportes são praticados por pessoas com deficiência fora dos movimentos esportivos formais ${ }^{1}$. Para além das questões relativas à abordagem esportiva, as modalidades são organizadas em desportos coletivos e individuais e integram os programas de competição de cada grupo de deficiências. A respeito desse aspecto, modalidades coletivas são opção atrativa de prática esportiva para $\mathrm{PCDs}^{6}$, principalmente pelo aspecto pessoal e social, que contribui para interação com pessoas que se encontram nas mesmas condições físicas.

Pensando em produção científica algumas críticas persistem até os dias atuais acerca dos estudos desenvolvidos com PCDs no contexto esportivo, principalmente em relação à questões metodológicas e qualidade das pesquisas aplicadas ${ }^{7}$. Contrariamente, evidências científicas em modalidades esportivas para PCDs tem crescido de forma constante, sobretudo em algumas áreas do conhecimento ${ }^{8}$. Face ao interesse nas lacunas existentes na produção científica acerca do esporte para PCDs, o objetivo do presente trabalho foi revisar a produção científica a respeito de modalidades esportivas coletivas para PCDs e sugerir direcionamentos para estudos futuros.

\section{Métodos}

\section{Mecanismos de busca}

Seguiram-se as recomendações PRISMA (Preferred Reportingltems for Systematic Reviews and Meta-Analysis) que orientam a redação de revisões sistemáticas ${ }^{9}$. Estudos para a presente revisão foram identificados a partir de busca sistemática em três bancos de dados (PubMed,SportDiscuss e Scopus), de maneira isolada e/ou combinada. Para a estratégia de buscacompleta foi elaborada a seguinte frase de pesquisa: ("Football 5-a-side" OR "Football 7-a-side" OR "Goalball" OR "Sitting volleyball" OR "Wheelchairbasketball" OR "Wheelchairrugby" OR "Wheelchairhandball" OR "amputee soccer" OR "amputee football" OR "power soccer" OR "powerchair football" OR "Power Soccer Wheelchair" OR "quadrugby" OR "Futebol de cinco" OR "Futebol de 5" OR "Futebol de 7" OR "Futebol de PC" OR "Goalball" OR "voleibol sentado" OR "vôlei sentado" OR "basquete em cadeira de rodas" OR "rugby em cadeira de rodas" OR "handebol em cadeira de rodas" OR "futebol para amputados" OR "futebol em cadeira de rodas"). A pesquisa foi limitada para os idiomas inglês e português, e à estudos publicados até 19 de janeiro de 2018

\section{Critérios de seleção}

Os títulos e resumos foram inicialmente digitalizados e todos artigos duplicados e fora do âmbito deste estudo foram excluídos. Os estudos foram incluídos se eles estavam publicados ou "impress" antes da data de busca. Os resumos foram avaliados e artigos foram excluídos se (a) os participantes não eram atletas com deficiência; $(b)$ esportes individuais ou em duplas; (c) os artigos não relataram quaisquer informações acerca das modalidades coletivas, $(d)$ eles eram artigos de revisão, opinião, relatos de caso ou estavam em idioma diferente do exposto acima. As versões integrais dos estudos foram recuperadas e analisadas segundo os mesmos critérios. Dois revisores avaliaram de forma independente os títulos e resumos antes de decidirem a respeito da inclusão dos estudos. Um dos revisores extraiu os dados relevantes de cada artigo incluído, enquanto o segundo revisor conferiu a extração dos dados.

\section{Sumário de resultados}

Os trabalhos incluídos foram sumarizados e analisados em relação a frequência absoluta (geral) e relativa (modalidade), sendo subdivididos em 13 áreas de concentração dos estudos (Tabela 1). Cabe ressaltar que os assuntos abordados em cada estudo poderiam se enquadrar em mais de uma área de investigação.

Tabela 1

Descrição e definição das áreas de estudo/temáticas sumarizadas para revisão sistemática

\begin{tabular}{|c|c|}
\hline $\begin{array}{c}\text { Área de } \\
\text { estudo/temática }\end{array}$ & Definições \\
\hline Biomecânica & $\begin{array}{l}\text { Características do aparelho locomotor, análise do } \\
\text { movimento humano em função das características } \\
\text { anatômicas e fisiológicas do corpo humano e das leis } \\
\text { da física }^{10}\end{array}$ \\
\hline $\begin{array}{l}\text { Classificação } \\
\text { esportiva }\end{array}$ & $\begin{array}{l}\text { Processo em que um único grupo de entidades (ou } \\
\text { unidades) são ordenados em vários grupos menores } \\
\text { (ou classes) com base em propriedades observáveis } \\
\text { que têm em comum }\end{array}$ \\
\hline $\begin{array}{l}\text { Caracterização } \\
\text { da modalidade }\end{array}$ & Características especificas de cada modalidade ${ }^{12}$ \\
\hline $\begin{array}{l}\text { Analise de } \\
\text { desempenho }\end{array}$ & $\begin{array}{l}\text { Variáveis presentes em situações de treino e } \\
\text { competição utilizadas para melhorar o desempenho }_{\text {esportivo }}{ }^{13}\end{array}$ \\
\hline $\begin{array}{l}\text { Saúde e } \\
\text { qualidade vida }\end{array}$ & $\begin{array}{l}\text { Aspectos relacionados preocupação com a modificação } \\
\text { e o Aprimoramento dos componentes da qualidade de } \\
\text { vida, principalmente no estado do organismo quando } \\
\text { funciona otimamente sem evidência de doenças } \\
\text { secundárias }^{14}\end{array}$ \\
\hline $\begin{array}{l}\text { Nutrição no } \\
\text { esporte }\end{array}$ & $\begin{array}{l}\text { Práticas alimentares dos atleta para suportar atividades } \\
\text { de treinamento, suplementação e aspectos relativos à } \\
\text { composição corporal }^{15}\end{array}$ \\
\hline $\begin{array}{l}\text { Psicologia do } \\
\text { esporte }\end{array}$ & $\begin{array}{l}\text { Fatores comportamentais que influenciam e são } \\
\text { influenciados pela participação e desempenho no } \\
\text { esporte, exercício e atividade física }{ }^{16}\end{array}$ \\
\hline Fisiologia & $\begin{array}{l}\text { Funções e processos de fisiológicos relacionados aos } \\
\text { diferentes sistemas do corpo humano em resposta ao } \\
\text { exercício físico e/ou desempenho esportivo }\end{array}$ \\
\hline Fisioterapia & $\begin{array}{l}\text { Tratamento de condições adversas do sistema } \\
\text { musculoesquelético, tais como lesões decorrentes da } \\
\text { prática esportiva }^{18}\end{array}$ \\
\hline $\begin{array}{l}\text { Treinamento } \\
\text { físico }\end{array}$ & $\begin{array}{l}\text { Estudos que abordam o processo organizado e } \\
\text { sistemático de aperfeiçoamento físico, nos seus } \\
\text { aspectos morfológicos e funcionais, impactando na a } \\
\text { capacidade de execução de tarefas esportivas }^{19}\end{array}$ \\
\hline $\begin{array}{l}\text { Tecnologia no } \\
\text { esporte }\end{array}$ & $\begin{array}{l}\text { Desenvolvimento tecnológicos atuais em dispositivos } \\
\text { auxiliares utilizados pelos atletas, tais como cadeira de } \\
\text { rodas, próteses, muletas, vestuário e etc }{ }^{20}\end{array}$ \\
\hline $\begin{array}{c}\text { Teste e } \\
\text { avaliações }\end{array}$ & $\begin{array}{l}\text { Desenvolvimento testes e avaliações específicos para } \\
\text { cada modalidade esportiva }{ }^{21}\end{array}$ \\
\hline $\begin{array}{l}\text { Aspectos Sociais } \\
\text { do esporte }\end{array}$ & $\begin{array}{l}\text { Aspectos relacionados ao fenômeno esporte para } \\
\text { atletas com deficiência, enfocando discussões acerca } \\
\text { de inclusão, integração e exclusão, assim como } \\
\text { aspectos históricos }\end{array}$ \\
\hline
\end{tabular}

\section{Resultados}

O processo de pesquisa, seleção e inclusão é ilustrado na Figura 1. A pesquisa revelou o total de 941 estudos, que foram reduzidos a 348 trabalhos após exclusão dos estudos duplicados (419 estudos) e estudos que não atenderam os critérios de inclusão (174 estudos). A lista com os trabalhos incluídos na presente revisão encontram-se em anexo/material suplementar. 


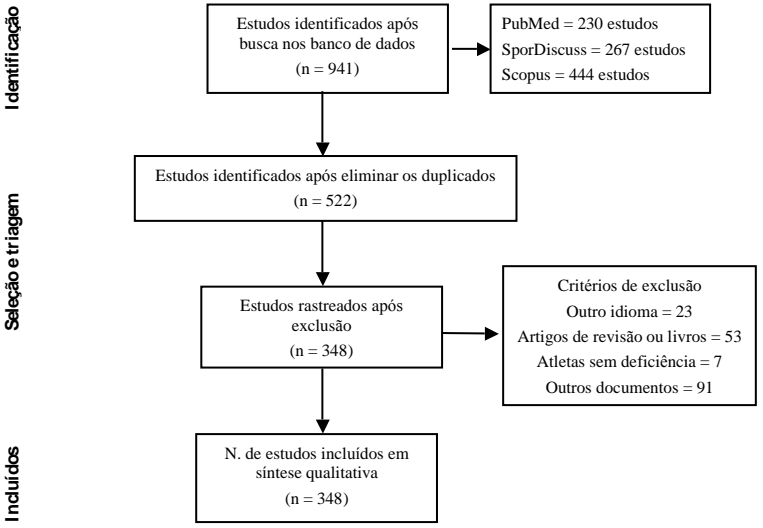

Figura 1. Procedimentos de identificação, triagem e inclusão de artigos na presente pesquisa.

De forma geral, Basquete e Rugby foram os esportes coletivos em cadeira de rodas que apresentaram a maior frequência de estudos identificados após síntese qualitativa (Tabela 2). Em outras modalidades para deficientes físicos, o Futebol para Amputados foi o que apresentou maior quantitativo de estudos. No caso de esportes para pessoas com deficiência visual, goalball apresentou maior frequência de estudos.

Tabela 2

Frequência relativa dos estudos com modalidades coletivas para PCDs $(n=353)$

\begin{tabular}{cccc}
\hline Modalidade & $\begin{array}{c}\text { Ano de } \\
\text { criação }\end{array}$ & $\mathrm{n}$ & $\%$ \\
\hline Basquete em cadeira de rodas $^{\mathrm{a}}$ & $1944-1945$ & 183 & $52 \%$ \\
Futebol de cinco $^{\mathrm{a}}$ & 1950 & 8 & $2 \%$ \\
Futebol de sete & 1978 & 1 & $0 \%$ \\
Futebol em cadeira de rodas $_{\text {Futebol para Amputados }}$ & 1970 & 2 & $1 \%$ \\
Goalball $^{\mathrm{a}}$ & 1980 & 22 & $6 \%$ \\
Handebol em cadeira de rodas $_{\text {Rugby em cadeira de rodas }}^{\mathrm{a}}$ & 1946 & 26 & $7 \%$ \\
Voleibol sentado & 1970 & 2 & $1 \%$ \\
Total & 1956 & 14 & $27 \%$ \\
\hline
\end{tabular}

a. Indica agrupamento da quantidade de estudos com outra modalidade similar.

A tabela 3 apresenta os resultados do agrupamento dos estudos em áreas de investigação. Genericamente, os estudos analisados na presente revisão tem se interessado em investigar à análise de desempenho esportivo $(n=129$; $21,6 \%)$, aspectos fisiológicos ( $n=95 ; 15,9 \%)$ e psicológicos $(\mathrm{n}=57 ; 9,5 \%)$

Tabela 2

Distribuição das áreas de estudo por modalidade analisada

\begin{tabular}{|c|c|c|c|c|c|c|c|c|c|c|}
\hline $\begin{array}{l}\text { Área de } \\
\text { estudo }\end{array}$ & $\begin{array}{l}\text { Basquete em } \\
\text { cadeira de } \\
\text { rodas }\end{array}$ & $\begin{array}{l}\text { Futebol } \\
\text { de cinco }\end{array}$ & $\begin{array}{l}\text { Futebol } \\
\text { de sete }\end{array}$ & $\begin{array}{l}\text { Futebol em } \\
\text { cadeira de } \\
\text { rodas }\end{array}$ & $\begin{array}{l}\text { Futebol para } \\
\text { amputados }\end{array}$ & Goalball & $\begin{array}{l}\text { Handebol } \\
\text { em cadeira } \\
\text { de rodas }\end{array}$ & $\begin{array}{l}\text { Rugby em } \\
\text { cadeira de } \\
\text { rodas }\end{array}$ & $\begin{array}{l}\text { Voleibol } \\
\text { Sentado }\end{array}$ & $\begin{array}{l}\text { Total } \\
\text { n (\%) }\end{array}$ \\
\hline Biomecânica & 21 & 1 & 0 & 0 & 0 & 0 & 1 & 17 & 0 & $\begin{array}{c}40 \\
(6,7 \%)\end{array}$ \\
\hline $\begin{array}{l}\text { Classificação } \\
\text { esportiva }\end{array}$ & 23 & 0 & 0 & 0 & 0 & 0 & 1 & 17 & 0 & $\begin{array}{c}41 \\
(6,9 \%)\end{array}$ \\
\hline $\begin{array}{l}\text { Caracterização da } \\
\text { modalidade }\end{array}$ & 14 & 3 & 1 & 1 & 7 & 8 & 1 & 3 & 1 & $\begin{array}{c}39 \\
(6,5 \%)\end{array}$ \\
\hline $\begin{array}{l}\text { Analise de } \\
\text { desempenho }\end{array}$ & 60 & 2 & 1 & 1 & 7 & 11 & 1 & 36 & 10 & $\begin{array}{c}129 \\
(21,6 \%)\end{array}$ \\
\hline $\begin{array}{l}\text { Saúde e qualidade } \\
\text { vida }\end{array}$ & 7 & 1 & 0 & 0 & 5 & 4 & 0 & 3 & 1 & $\begin{array}{c}21 \\
(3,5 \%)\end{array}$ \\
\hline Nutrição no esporte & 11 & 1 & 0 & 0 & 4 & 3 & 0 & 7 & 0 & $\begin{array}{c}26 \\
(4,4 \%)\end{array}$ \\
\hline $\begin{array}{l}\text { Psicologia do } \\
\text { esporte }\end{array}$ & 33 & 0 & 0 & 1 & 3 & 3 & 0 & 14 & 3 & $\begin{array}{c}57 \\
(9,5 \%)\end{array}$ \\
\hline Fisiologia & 49 & 2 & 0 & 0 & 5 & 5 & 0 & 31 & 3 & $\begin{array}{c}95 \\
(15,9 \%)\end{array}$ \\
\hline Fisioterapia & 17 & 3 & 1 & 0 & 6 & 2 & 1 & 5 & 0 & $\begin{array}{c}35 \\
(5,9 \%)\end{array}$ \\
\hline Treinamento físico & 22 & 1 & 0 & 0 & 7 & 1 & 0 & 10 & 3 & $\begin{array}{c}44 \\
(7,4 \%)\end{array}$ \\
\hline $\begin{array}{l}\text { Tecnologia no } \\
\text { esporte }\end{array}$ & 7 & 0 & 0 & 0 & 2 & 0 & 0 & 7 & 0 & $\begin{array}{c}16 \\
(2,7 \%)\end{array}$ \\
\hline Teste e avaliações & 19 & 0 & 0 & 0 & 1 & 1 & 2 & 8 & 4 & $\begin{array}{c}35 \\
(5,9 \%)\end{array}$ \\
\hline $\begin{array}{l}\text { Aspectos Sociais } \\
\text { do esporte }\end{array}$ & 14 & 2 & 0 & 1 & 4 & 2 & 0 & 7 & 1 & $\begin{array}{c}31 \\
(5,2 \%) \\
\end{array}$ \\
\hline $\mathrm{n}^{\circ}$ de estudos & 183 & 8 & 1 & 2 & 22 & 26 & 2 & 95 & 14 & \\
\hline
\end{tabular}

Os resultados específicos por modalidade sinalizam para aspectos diferentes de investigação. Nesse caso, estudos com Basquete e Rugby em cadeira de rodas tem se interessado para aspectos de desempenho esportivo, fisiológicos, classificação esportiva e biomecânico. No futebol para amputados, os estudiosos têm direcionado os interesses nas pesquisas que abordam a caracterização da modalidade, as análises de desempenho esportivo e aspectos relacionados à fisioterapia. Já em relação às deficiências visuais, o goalball apresentou maior quantidade de estudos, quando comparado com o futebol de cinco. Os resultados mostraram que as publicações direcionadas para modalidade goalball relacionam-se com os aspectos de desempenho esportivo e caracterização da modalidade.

\section{Discussão}

O principal objetivo do estudo foi revisar a produção científica a respeito de modalidades esportivas coletivas para PCDs e sugerir direcionamentos para estudos futuros. Nossos principais achados foram que (a) análise de desempenho esportivo, aspectos fisiológicos e psicológicos foram as principais áreas de concentração dos estudos e (b) nós encontramos poucos estudos relativos a saúde e qualidade vida, nutrição esportiva, fisioterapia, tecnologia no esporte, teste e avaliações e nos aspectos sociais do esporte adaptado. Em adição, Basquete, Rugby em cadeira de rodas, Goalball e Futebol para Amputados foram os esportes coletivos que apresentaram maior frequência de estudos após síntese qualitativa. Resultados apresentados no presente estudo revelam que pesquisas com as modalidades Futebol de cinco, Futebol de sete, Futebol em cadeira de rodas e Handebol em cadeira de rodas são escassas. No decorrer da história das PCDs as praticas esportivas evoluíram do componente médico-terapêutico para 0 desporto de rendimento ${ }^{23}$, procurando a integração do atleta e sua reabilitação social ${ }^{24}$. Esportes competitivos para pessoas com deficiência (PCDs) têm crescido ao longo das últimas décadas $^{25,26}$, o que contribui para que os investigadores desenvolvam estudos com a vertente do esporte de rendimento, o que explica os resultados da presente revisão.

O processo de análise de desempenho esportivo têm contribuido na compreensão do perfil de exigências específicas de cada modalidade principalmente nas situações 
de treino e de competição ${ }^{13}$. Nas modalidades coletivas o comportamento requerido aos atletas é regulado pela adaptação às novas situações mediante a aplicação das habilidades já adquiridas, modificando a estrutura da habilidade e sua posterior reorganização em nível superior de complexidade ${ }^{27}$. Analisando pelo prisma das modalidades coletivas para PCDs, a reorganização da complexidade é parte do processo de ensino- apresendizagem-treinamento. Por exemplo, no caso das deficiências físicas adquiridas, o primeiro nível de complexidade a ser vencido relaciona-se com o deslocamento em quadra utilizando-se cadeira de rodas $^{26}$ ou mesmo muletas ${ }^{12}$. Em modalidades para deficientes visuais as questões de percepção espacial e auditiva dinâmicas emergem como as fontes de reorganização do movimento ${ }^{28}$.

Treinadores e pesquisadores das ciências dos esportes geralmente usam análise de desempenho para avaliar quatro principais determinantes de desempenho: as demandas físicas, psicológicas, técnicas e cognitivas do esporte ${ }^{29}$. Em nosso estudo identificamos que os pesquisadores tem direcionado as investigações para os aspectos técnicos, principalmente no basquete em cadeira de rodas ${ }^{30-32}$, rugby em cadeira de rodas ${ }^{33,34}$ e goalball ${ }^{35,36}$. No caso das análises das demandas físicas, os estudos tem fornecido visão quantitativa das relações entre cargas externas (e. g. distância percorrida, velocidades alcançadas, relações de pausaesforço, etc.) e internas (e. g. respostas da frequência cardíaca, lactato sanguíneo, percepção de esforço e etc) em situações de treinamento ou competição principalmente no basquete em cadeira de rodas ${ }^{37,38}$, rugby em cadeira de rodas $^{39-41}$ e no futebol para amputados ${ }^{42,43}$. Análises táticas geralmente fornecem resumo dos resultados de desempenho (por exemplo, posicionamento de defesa, distribuição de arremessos, etc.), praticas frequentes no basquete ${ }^{37,44}$ e rugby em cadeira de rodas ${ }^{45,46}$ e no goalbaal ${ }^{47,48}$

Outro ponto a ser destacado nas análises de desempenho esportivo se concentra na escolha dos jogadores que vão constituir a equipe. No esporte para PCDs esse é um fato delicado, principalemente porque a maioria dos indivíduos com deficiência não desejavam se tornar deficientes. Adicionalmente cada modalidade é dotada de especificidade que influenciam o planejamento do treinamento ${ }^{49,50}$. Exemplo disso acontece nas modadalides em cadeiras de rodas, nas quais em função da classificação esportiva funcional os atletas apresentam diferentes potenciais funcionais, existindo grupos para desempenhar ações especificas (e. g. bloqueios nas situações ou carregar a bola nas situações de ataque ${ }^{26}$. A esse respeito, nós encontramos relações existentes entre os estudos de desempenho esportivo e classificação esportiva, principalmente em modalidades em cadeira de rodas. Muitos estudos se concentraram nas relações entre testes de campo e classses funcionais ${ }^{51-54}$, desempenho em situações de treinamento/competição $0^{41,45,46,55}$ e classificação funcional e na influência da força de tronco de diferentes graus de deficiência no desempenho esportivo ${ }^{56-58}$.

Em conjunto com a análise de desempenho esportivo, o mapeamento das características fisiológicas de atletas com diferentes deficiências tem contribuído para compreensão das demandas durante competição e treinamento ${ }^{49,59,60}$. Especificamente em atletas com deficiência, as questões fisiológicas emergem como prioritárias principalmente no que diz respeito à compreensão das respostas agudas e crônicas provenientes do treinamento desportivo ${ }^{49,50}$. Um exemplo disso é a preocupação dos pesquisadores em investigar as respostas de parâmetros fisiológicos em atletas com lesão medular $^{50}$, principalmente em virtude da perda de função autonômica abaixo do nível da lesão. Dentre as principais implicações desse fato estão a diminuição da frequência cardíaca de pico ${ }^{59,60}$, diminuição das concentrações plasmáticas de adrenalina e noradrenalina em repouso e após o exercício ${ }^{61,62}$ e diminuição da atividade neuronal simpática ${ }^{49,60}$. No caso de outras deficiências físicas, como pessoas com amputação de membros inferiores, as alterações fisiológicas determinantes para o desempenho esportivo se concentram no aumento na frequência cardíaca durante a deambulacão com muletas em virtude do menor retorno venoso $0^{42,63}$ e das diferenças entre os níveis de amputação e gasto calórico ${ }^{42,64}$. Contrariamente ao que ocorre com deficiências física, a preocupação com os aspectos fisiológicos não acontece quando se avalia pessoas com deficiência visual uma vez que as alterações provenientes da deficiência estão concentradas nos sentidos do corpo humano, em especial na visão ${ }^{5,65}$

Os aspectos relativos à psicologia do esporte aparecem em nossa pesquisa como uma das principais áreas de concentração dos estudos com modalidades coletivas para PCDs. Para competir, além de condição física, técnica e tática adequada, os atletas com deficiência necessitam de controle de diversas variáveis psicológicas para alcançarem o sucesso ${ }^{66}$. Em linhas gerais, o ambiente esportivo é como uma montanha-russa emocional, ou seja, intensa, comovente e constante. A avaliação de atletas com deficiência no aspecto psicológico é procedimento complexo em função da heterogeneidade do grupo, de seu perfil e da influência de inúmeras variáveis ${ }^{16,67}$. Atletas com deficiência devem usar métodos psicológicos para alcançar habilidades mentais, aprendendo a se autorregular por meio da definição de metas, aprimorando e mantendo motivação para pratica esportiva ${ }^{16,67}$. Entretanto, é comum pensar que atletas com deficiência experimentam baixa autoestima pois sua deficiência pode limitar o sucesso no esporte ${ }^{68}$. Contrariamente a esse ponto, a literatura vem demonstrando que praticas esportivas contribuem para inserção dos deficientes na sociedade ${ }^{24,25,68}$ auxiliando na melhora da qualidade de vida ${ }^{14}$. Características como autodeterminação, autoconceito, resiliência $e$ estabilidade emocional são desejáveis em atletas com deficiência, principalmente porque essas qualidades muitas vezes têm impacto indireto na qualidade de treinamento ${ }^{16,69}$. Esses conceitos psicológicos positivos e fatores de personalidade também são importantes para o sucesso esportivo ${ }^{69}$. Não é incomum que pessoas com deficiência experimentem sentimentos de impotência ${ }^{67}$. Por exemplo, é difícil imaginar que algum atleta possa treinar e competir em nível ótimo de rendimento durante muitos anos se eles se sentem impotentes, não valorizam quem são. Esse atleta seria propenso a ansiedade, estresse e depressão, e risco de se tornar desmotivado. Dessa maneira, intervenções utilizando os conceitos de psicologia do esporte podem contribuir para melhor controle emocional e mental dos atletas.

Nosso estudo indicou a necessidade de produção científica nas áreas de saúde e qualidade vida, fisioterapia, nutrição esportiva, tecnologia no esporte, teste e avaliações e nos aspectos sociais do esporte adaptado. Pensando que atletas com deficiência podem apresentar condições secundárias de saúde (e. g. hipertensão, diabetes, infecções e etc), pesquisas são necessárias para avançar a compreensão de questões relacionadas ao efeito da atividade física e exercício físico na saúde e prevenção de doenças entre $\mathrm{PCDs}^{70}$. À medida que PCDs vivem mais tempo, a necessidade de abordar questões de saúde a longo prazo e o risco de incapacidade secundária devem receber maior atenção ${ }^{70}$. Ademais, o treinamento esportivo deve ser estudado desde a perspectiva da prevenção de doenças ${ }^{70}$, ao mesmo tempo que pode atenuar o risco de lesões musculoesqueléticas ${ }^{71}$, reforçando a necessidade de estudos e intervenções na área da fisioterapia esportiva.

Considerando que o conselho dietético com base em princípios gerais de saúde pode ter sido suficiente para PCDs, existe atualmente a necessidade de apoio nutricional especializado para alcançar objetivos de treinamento e competição $^{15}$. Para atletas com PCDs os requisitos de nutrientes devem ser avaliados em relação às diferenças nos níveis de atividade, processos metabólicos alterados, medicamentos crônicos, modos variados de alimentação e tipo de deficiência. Especificamente, alguns tipos de deficiência têm capacidades fisiológicas e físicas alteradas ${ }^{15,72}$ e muitas modalidades coletivas têm demandas fisiológicas únicas, como basquete em cadeira de rodas ${ }^{73,74}$, goalball ${ }^{75,76}$ rugby de cadeira de rodas ${ }^{77,78}$ e futebol para amputados ${ }^{79,80}$ Por outro lado, a massa corporal de PCDspode necessitar de ajuste das diretrizes usuais, principalmente nos casos de avaliação em relação à composição corporal de tetraplégicos ou amputados ${ }^{15}$. Todas essas razões confirmam a necessidade de pesquias na área da nutrição esportiva para auxiliar os profissionais que trabalham com atletas com deficiência.

Nos esportes para PCDs, tecnologia e testes são utilizados como meio de aprimoramento do desempenho 
esportivo $^{20}$, tendo como foco os equipamentos utilizados pelos atletas para competir ou treinar ${ }^{29}$. Em nosso estudo, equipamentos como cadeiras de rodas personalizadas ${ }^{81-83}$, muletas canadenses ${ }^{84}$ são exemplos de desenvolvimentos tecnológicos para esportes em cadeira de rodas e futebol para amputados. Entretanto, os estudos também se direcionaram para o desenvolvimento de roupas esportivas ${ }^{85}$, equipamentos para realização de testes ${ }^{86,87}$ e análises de tempo-movimento durante competições ${ }^{40,41,88}$.

Em relação aos aspectos sociais, nosso trabalho revelou a necessidade de mais estudos serem desenvolvidos nessa área de pesquisa. De maneira geral, aspectos sociais auxiliam na comprenção do papel social que a deficiência pode ter na formação dos contextos de treinamento ${ }^{68}$. É comum no caso de atletas com deficiência o fato dele (atleta) trabalhar em tempo integral, o que limita oportunidades de treinamento regular com seus companheiros de equipe, além de em muitos casos precisar percorrer longas distâncias para aproveitar as oportunidades para competir ${ }^{15}$. Adicionalmente é necessário destacar que 0 ambiente esportivo oferece diversas experiências sociais, além de possibilitar oportunidades para socialização entre os participantes da equipe $^{89}$. Múltiplos fatores complexos provavelmente contribuem para disparidades sociais, incluindo barreiras estruturais, socioeconômicas e atitudes que impedem a ampla participação de PCDs no convívio social ${ }^{4,22}$.

Interessantemente, nossos resultados indicaram as modaldidades basquete, rugby em cadeira de rodas, goalball e futebol para amputados como os esportes coletivos com maior percentual de trabalhos recuperados após busca. Analisando historicamente, basquete em cadeira de rodas são modalidade mais antigas das que fizeram parte do presente estudo, jutificando assim nossos resultados. O que foi supreendete em nosso resultado foi encontrar modalidades como rugby em cadeira de rodas e futebol para amputados com estudos mais frequentes. Esse fato pode ser explicado de duas maneira: (a) níveis de lesão mais comprometidos no rugby em cadeira de rodas, o que desperta interesse de profissionais em compreender as respostas do exercício nesse grupo ${ }^{26,59}$ e $(b)$ o futebol para amputados tem se organizado para fazer parte do grupo de modalidades que compoem os Jogos Paralímpicos ${ }^{12}$. Além disso, as modalidades esportivas Futebol de cinco, Futebol de sete, Futebol em cadeira de rodas e Handebol em cadeira de rodas apresentaram percentual inferior de trabalhos publicaos, reforçando a necessidade de desenvolvimento de pesquisas com essas modalidades em todas áreas de conhecimento.

\section{Limitação de estudo}

O número de pessoas com deficiência envolvidas em esportes está aumentando constantemente em todo o mundo com esportes organizados para atletas com deficiência divididos em grupos de deficiência ou seja, esportes para surdos, para deficientes físicos, para deficientes visuais e pessoas com deficiência intelectual. Dessa maneira, os achados do presente estudo são limitados à modalidades coletivas disputadas por deficientes físicos e visuais, não representando a totalidade em relação aos grupos de deficiência. Sugerimos que outros estudos sejam realizados para abordar as questões esportivas relativas aos deficientes auditivos e intelectuais. Adionalmente, estudos futuros devem investigar as tematicas específicas em cada uma das áreas de concentração sugeridas na presente revisão.

\section{Conclusão}

Concluímos que estudos recuperados na presente revisão se concentram na análise de desempenho esportivo, aspectos fisiológicos e psicológicos, principalmente nas modalidades basquete em cadeira de rodas, rugby em cadeira de rodas, futebol para amputados e goaball. Sugerimos que pesquisadores avançem nos conhecimentos fisiológicos, de desempenho esportivo e de psicologia do esporte em todas modalidades investigadas, mas que também desenvolvam estudos relativos a saúde e qualidade vida, nutrição esportiva, fisioterapia, tecnologia no esporte, teste e avaliações e aspectos sociais do esporte para PCDs.

\section{Referências}

1. Araújo PF. Desporto adaptado no Brasil. São Paulo: Phorte Editora 2011.

2. Howe PD, Jones C. Classification of disabled athletes: (dis) empowering the paralympic practice community. Sociology of Sport Journal. 2006;23:29-46.

3. Hutzler Y. Adapted Physical Activity and Sport in Rehabilitation. In: Stone $\mathrm{JH}$, Blouin $\mathrm{M}$, editors. International Encyclopedia of Rehabilitation2010.

4. Simim MAM. Exercício, esporte e inclusão: a formação do profissional de educação física e o esporte adaptado. In: Noce F, editor. O profissional de educação física na área da saúde. Belo Horizonte: EEFFTO; 2014. p. 71-6.

5. Winnick J. Educação Física e Esportes Adaptados. Barueri/SP: Manole; 2004

6. Borges M, Costa e Silva AA, Faria FR, Godoy PS, Calegari DR, Araujo PF, et al. Handebol em Cadeira de Rodas: fundamentos da modalidade. Conexões. 2015;13(3):195-212.

7. Mauerberg-deCastro E, Campbell DF. The global reality of the Paralympic Movement: challenges and opportunities in disability sports. Motriz. 2016;22(3):111-23.

8. Paulson T, Goosey-Tolfrey V. Current Perspectives on Profiling and Enhancing Wheelchair Court Sport Performance. International Journal of Sports Physiology and Performance. 2016;12(3):275-86.

9. Moher D, Liberati A, Tetzlaff J, Altman DG, PRISMAGroup. Preferred Reporting Items for Systematic Reviews and Meta-Analyses: The PRISMA Statement. PLoS Med 2009;6(6):e1000097.

10. Amadio AC, Serrão JC. A Biomecânica em Educação Física e Esporte. Revista Brasileira de Educação Física e Esporte. 2011:25:15-24

11. Tweedy S, Howe D. Introduction to the Paralympic Movement. In Vanlandewijck YC, Thompson WR, editors. :0Bi:Handbook of Sports Medicine and Science - The Paralympic Athlete: Blackwell Publishing 2011. p. 3-30.

12. Simim MAM, Silva BVC, Mota GR. Futebol para amputados: aspectos técnicos, táticos e diretrizes para o treinamento. Revista Brasileira de Futsal e Futebol. 2015;7:246-54

13. O'Donoghue P. Research Methods for Sports Performance Analysis Oxon: Routledge; 2010.

14. Noce F, Simim MAM, de Mello MT. A Percepção de Qualidade de Vida de Pessoas Portadoras de Deficiência Física Pode ser Influenciada Pela Prática de Atividade Física? Revista Brasileira de Medicina do Esporte. 2009;15(3):174-8.

15. Broad E. Sports Nutrition for Paralympic Athletes: Taylor \& Francis Group; 2010.

16. Samulski DM, Noce F, Costa VT. Mental preparation. In: Vanlandewijck YC, Thompson WR, editors. Handbook of Sports Medicine and Science - The Paralympic Athlete: Blackwell Publishing 2011. p. 198-213.

17. Powers S, Howley E. Fisiologia do Exercício - Teoria e Aplicação ao Condicionamento e ao Desempenho: Manole; 2014. 672 p.

18. Silva A, Vital R, de Mello MT. Atuação da fisioterapia no esporte paralímpico. Revista Brasileira de Medicina do Esporte. 2016;22(2):15761.

19. Roschel H, Tricoli V, Ugrinowitsch C. Treinamento físico: considerações práticas e científicas . . Revista Brasileira de Educação Física e Esporte. 2011;25:53-65.

20. Burkett B. Technology in Paralympic sport: performance enhancement or essential for performance? British Journal of Sports Medicine. 2010;44(3):215-20

21. Gorla JI, Campana MB, Oliveira LZ. Teste e Avaliação em Esporte Adaptado. São Paulo: Phorte Editora; 2009. 224 p.

22. Howe D. Sociology. In: Vanlandewijck YC, Thompson WR, editors. Do $\mathrm{B}$ - Handbook of Sports Medicine and Science - The Paralympic Athlete: Blackwell Publishing; 2011. p. 102-15.

23. Brittain I. The Paralympic Games: from a rehabilitation exercise to elite sport (and back again?). International Journal of Therapy and Rehabilitation. 2012;19(9):526-31.

24. Costa AM, Sousa SB. Educação Física e Esporte Adaptado: história, avanços, retrocessos em relação aos princípios da integração/inclusão e perspectivas para o Século XXI Revista Brasileira de Ciências do Esporte. 2004;25(3):27-42

25. Nyland JA. The paralympic movement: addition by subtraction. Journal of Orthopaedic \& Sports Physical Therapy. 2009;39(4):243-5

26. Costa e Silva AA, Marques RFR, Pena LGS, Molchansky S, Borges M, Campos LFCC, et al. Esporte adaptado: abordagem sobre os fatores que influenciam a prática do esporte coletivo em cadeira de rodas. Revista Brasileira de Educação Física e Esporte. 2013;27(4):679-87.

27. Tani G. Processo adaptativo em aprendizagem motora: o papel da variabilidade. Revista Paulista de Educação Física. 2000;Supl. 3:55-61.

28. Tosim A, Rodrigues GM, Mendonça MAB. Seleção da percepção auditiva e tátil em atletas de goalball em situação defensiva de jogo. auditiva e tátil em atletas de goalball em situação defensiva de jogo

29. Fliess-Douer O, Mason B, Katz L, Raymond So C. Sport and technology. In: Vanlandewijck YC, Thompson WR, editors. Training and Coaching the Paralympic Athlete: Wiley Blackwell; 2016. p. 150-71.

30. Goosey-Tolfrey V, Butterworth D, C. M. Free throw shooting technique of male wheelchair basketball players. Adapted Physical Activity Quarterly. 2002;19(2):238-50.

31. Malone LA, Gervais PL, Steadward RD. Shooting mechanics related to player classification and free throw success in wheelchair basketball. Journal of Rehabilitation Research and Development. 2002;39(6):701-9.

32. Schwark BN, Mackenzie SJ, Sprigings EJ. Optimizing the Release Conditions for a Free Throw in Wheelchair Basketball. Journal of Applied Biomechanics 2004;20(2):153-66.

33. Berzen J, Hutzler $Y$. Evaluating performance progression in beginner wheelchair rugby. European Journal of Adapted Physical Activity. 2012;5(1):53-64. 
34. Borren GL, Gooch SD, Ingram B, Jenkins A, Dunn J. Classification Efficiency in Wheelchair Rugby: Throwing Analysis. IFAC Proceedings Volumes. 2014;47(3):4772-7.

35. Bowerman $S$, Davis R, Ford S, Nichols D. Phases of movement of goalball throw related to ball velocity. Insight. 2011;4:153-9.

36. Stamou E, Theodorakis Y, Kokaridas D, Perkos S, Kessanopoulou M. The effect of self-talk on the penalty execution in goalball. British Journal of Visual Impairment. 2007;25(3):233-47.

37. Bloxham LA, Bell GJ, Bhambhani Y, Steadward RD. Time motion analysis and physiological profile of Canadian World Cup wheelchair basketball players. Sports Medicine Training and Rehabilitation. 2001;10(3):183-97.

38. de Witte AM, Hoozemans MJ, Berger MA, van der Woude LH, Veeger $\mathrm{DH}$. Do field position and playing standard influence athlete performance in wheelchair basketball? Journal of Sports Sciences. 2016;34(9):81120.

39. Goosey-Tolfrey VL, Mason B, Burkett BT. The Role Of The Velocometer As An Innovative Tool For Paralympic Coaches To Understand Wheelchair Sporting Training And Interventions To Help Optimise Performance. Sports Technology. 2012;5(1-2):20-8.

40. Sarro KJ, Silvatti AP, Aliverti A, Barros RML. Tracking of wheelchair rugby players in the 2008 Demolition Derby final. Journal of Sports Sciences. 2010;28(2):193-200.

41. Rhodes JM, Mason BS, Perrat B, Smith MJ, Malone LA, Goosey-Tolfrey V. Activity profiles of elite wheelchair rugby players during competition. International Journal of Sports Physiology and Performance. 2015;10(3):318-24.

42. Simim MAM, Mota GR, Marocolo M, Silva BVC, Mello MT, Bradley PS. The Demands of Amputee Soccer Impair Muscular Endurance and Power Indices But Not Match Physical Performance. Adapted Physical Activity Quarterly. 2018;5:1-17.

43. Maehana H, Miyamoto A, Koshiyama K, Tanaka Yanagiya T, Yoshimura $M$. Profile of match performance and heart rate response in Japanese amputee soccer. Journal of Sports Medicine and Physical Fitness. 2017; [Epub ahead of print].

44. Gil-Agudo A, Del Ama-Espinosa A, Crespo-Ruiz B. Wheelchair basketball quantification. Physical medicine and rehabilitation clinics of North America. 2010;21(1):141-56.

45. Molik B, Lubelska E, Koxmol A, Bogdan M, Yilla AB, Hyla E. An examination of the international wheelchair rugby federation classification system utilizing parameters of offensive game efficiency. Adapted Physical Activity Quarterly. 2008;25(4):335-51.

46. Morgulec-Adamowicz N, Kosmol A, Bogdan M, Molik B, Rutkowska I, Bednarczuk G. Game efficiency of wheelchair rugby athletes at the 2008 paralympic games with regard to player classification. Human Movement. 2015;11(1):29-36.

47. Molik B, Morgulec-Adamowicz N, Kosmol N, Perkowski K, Bednarczuk G, Skowroński W, et al. Game Performance Evaluation in Male Goalball Players. Journal of Human Kinetics. 2015;22(48):43-51.

48. Morato MP, Furtado OLPC, Gamero DH, Magalhaes TP, Almeida JJG. Development and evaluation of an observational system for goalball match analysis. Revista Brasileira de Ciências do Esporte. 2017;39:398407.

49. Simim MAM, de Mello MT, Silva BVC, Rodrigues DF, Rosa JPP, Coutio $\mathrm{BP}$, et al. Load Monitoring Variables in Training and Competition Situations: A Systematic Review Applied to Wheelchair Sports. Adapted Physical Activity Quarterly. 2017;34(4):466-83.

50. Simim MAM, Silva RB, Candido RF, Silva BVC, Mendes EL, Mota GR. Desempenho esportivo em atletas de Rugby em cadeira de rodas: uma revisão sistemática. Revista Brasileira de Prescrição e Fisiologia do Exercício. 2013;7:244-52.

51. Cavedon V, Zancanaro C, Milanese C. Physique and Performance of Young Wheelchair Basketball Players in Relation with Classification. PLoS One. 2015:10(11):e0143621.

52. Gómez MA, Molik B, Morgulec-Adamowicz N, Szyman RJ. Performance analysis of elite women's wheelchair basketball players according to team-strength, playing-time and players' classification. International Journal of Performance Analysis in Sport. 2015;15(1):268-83.

53. Godoy PS, Borges M, Faria FR, Duarte E. Correlação entre classificação funcional, gênero e habilidades motoras de jogadores de handebol em cadeira de rodas. Revista Brasileira de Ciências do Esporte. 2017;39(4):424-32

54. Molik B, Laskin JJ, Kosmol A, Skucas K, Bida U. Relationship Between Functional Classification Levels and Anaerobic Performance of Wheelchair Basketball Athletes. Research Quarterly for Exercise and Sport. 2010;81:69-73.

55. Rhodes JM, Mason BS, Paulson TAW, Goosey-Tolfrey VL. A Comparison of Speed Profiles During Training and Competition in Elite Wheelchair Rugby Players. International Journal of Sports Physiology and Performance. 2017;12(6):777-82.

56. Özünlü N, Ergun N. Trunk balance assessment in wheelchair basketball players. Fizyoterapi Rehabilitasyon. 2012;23(1):44-50.

57. Altmann VC, Groen BE, Hart AL, Vanlandewijck YC, Keijsers NLW. Classifying trunk strength impairment according to the activity limitation caused in wheelchair rugby performance. Scandinavian Journal of Medicine \& Science in Sports. 2017; [Epub ahead of print]

58. Altmann VC, Groen BE, Hart AL, Vanlandewijck YC, van Limbeek J, Keijsers NLW. The impact of trunk impairment on performancedetermining activities in wheelchair rugby. Scandinavian Journal of Medicine \& Science in Sports. 2017;27(9):1005-14

59. Goosey-Tolfrey VL, Price M. Physiology of Wheelchair Sport. In: Goosey-Tolfrey VL, editor. Wheelchair Sport: A complete guide for athletes, coaches, and teachers: Human Kinetics; 2010. p. 47-62.

60. Bhambhani Y. Physiology of wheelchair racing in athletes with spinal cord injury. Sports Medicine. 2002;32(1):23-51.
61. Leicht CA, Bishop NC. The Effect of Acute and Chronic Exercise on Inflammatory Markers in SCl. In: Taylor JA, editor. The Physiology of Exercise in Spinal Cord Injury. Boston, MA: Springer US; 2016. p. $217-$ 31

62. Leicht CA, Goosey-Tolfrey V, Bishop NC. Spinal cord injury: known and possible influences on the immune response to exercise. . Exercise immunology review. 2013;19:144-63

63. Simim MAM, Silva BVC, Marocolo M, Mendes EL, Mello MT, Mota GR Anthropometric profile and physical performance characteristic of the Brazilian amputee football (soccer) team. Motriz. 2013;19(3):641-8.

64. Simim MAM, Bradley PS, Silva BVC, Mendes EL, Mello MT, Marocolo $M$, et al. The quantification of game-induced muscle fatigue in amputee soccer players. The Journal of sports medicine and physical fitness. 2017;57(6):766-72.

65. DePauw KP, Gavron SJ. Disability Sport. Champaign, IL: Human Kinetics; 2005.

66. Simim MAM, Costa VT, Samulski DM, Ferreira RM. Análise do estresse em atletas de futebol para amputados. Revista da Educação Física/UEM. 2011:21(2):237-44.

67. Samulski DM, Noce F. Perfil psicológico de atletas paraolímpicos brasileiros. Revista Brasileira de Medicina do Esporte. 2002;8(4):15766.

68. Martin J. Psychosocial Aspects of Youth Disability Sport. Adapted Physical Activity Quarterly. 2006;23:65-77.

69. Martin J. Mental preparation for the 2014 Winter Paralympic Games. Clinical Journal of Sport Medicine. 2012;22:70-3.

70. Cooper RA, Quatrano LA, Axelson PW, Harlan W, Stineman M, Franklin $\mathrm{B}$, et al. Research on physical activity and health among people with disabilities: a consensus statement. Journal of rehabilitation research and development. 1999;36(2):142-54.

71. Derman W, Runciman P, Schwellnus M, Jordaan E, Blauwet C Webborn N, et al. High precompetition injury rate dominates the injury profile at the Rio 2016 Summer Paralympic Games: a prospective cohort study of 51198 athlete days. British Journal of Sports Medicine. 2018;52(1):24-31.

72. Ribeiro SML, Silva RC, de Castro I, Tirapegui J. Assessment of nutritional status of active handicapped individuals. Nutrition Research. 2005;25(3):239-49.

73. Eskici G, Ersoy G. An evaluation of wheelchair basketball players' nutritional status and nutritional knowledge levels. Journal of Sports Medicine and Physical Fitness. 2016:56(3):259-68.

74. Ferro A, Garrido G, Villacieros J, Pérez J, Grams L. Nutritional Habits and Performance in Male Elite Wheelchair Basketball Players During a Precompetitive Period. Adapted Physical Activity Quarterly. 2017;34(3):295-310

75. Caliskan E, Pehlivan A, Erzeybek MS, Kayapinar FC, Agopyan A Yuksel $S$, et al. Body mass index and percent body fat in goalball and movement education in male and female children with severe visual impairment. Neurology, Psychiatry and Brain Research. 2011;17(2):3941 .

76. Gawlik K, Zwierzchowska A, Rosołek B. Evaluation of Lipid Metabolism and Nutritional Status in Male Goalball Players. Journal of Human Kinetics. 2015;12(48):141-7.

77. Gorla JI, Costa e Silva AA, Borges M, Tanhoffer RA, Godoy PS, Calegari DR et al. Impact of Wheelchair Rugby on Body Composition of Subjects With Tetraplegia: A Pilot Study. Archives of physical medicine and rehabilitation. 2016;97(1):92-6.

78. Black KE, Huxford J, Perry T, Brown RC. Fluid and sodium balance of elite wheelchair rugby players. International journal of sport nutrition and exercise metabolism. 2013;23(2):110-8.

79. Gomes AIS, Ribeiro BG, Soares EA. Nutritional profile of the Brazilian Amputee Soccer Team during the precompetition period for the world championship. Nutrition. 2006;22(10):989-95.

80. Gomes AIS, Ribeiro BG, Soares EA. Caracterização nutricional de jogadores de elite de futebol de amputados. Revista Brasileira de Medicina do Esporte. 2005;11(1):11-6

81. Berger MAM, Van Nieuwenhuizen M, Van Der Ent M, Van Der Zande M. Development of a new wheelchair for wheelchair basketball players in the Netherlands. Procedia Engineering. 2012;34:331-6.

82. Burton M, Subic A, Mazur M, Leary M. Systematic design customization of sport wheelchairs using the Taguchi method. Procedia Engineering. 2010;2(2):2659-65

83. Haydon DS, Pinder RA, Grimshaw PN, Robertson WSP. Elite wheelchair rugby: a quantitative analysis of chair configuration in Australia. Sports Engineering. 2016;19(3):177-84.

84. Ramazanoğlu N, Tatar Y, Camlıgüney AF, Uzun S, Cotuk HB. Effect of the use of Lofstrand crutches and prostheses on some gait parameters in amputee football players. Pamukkale Journal of Sport Science. 2013;3(3):59-65.

85. Çinarli S. Market research by means of a demand analysis for sports garments designed and produced for the amputated individuals. Teksti ve Konfeksiyon. 2012;22(3):185-9.

86. Faupin A, Gorce P, A. T. A wheelchair ergometer adaptable to the rearwheel camber. International Journal of Industrial Ergonomics. 2008;38(7-8):601-7.

87. van der Slikke RMA, Mason BS, Berger MAM, Goosey-Tolfrey VL. Speed profiles in wheelchair court sports; comparison of two methods for measuring wheelchair mobility performance. Journal of Biomechanics. 2017;65:221-5

88. Mason B, Lenton J, Rhodes J, Cooper R, Goosey-Tolfrey V. Comparing the activity profiles of wheelchair rugby using a miniaturised data logge and radio-frequency tracking system. BioMed Research International. 2014; Article ID 348048:8 pages.

89. Simim MAM, Silva BVC, Penna EM, Ferreira RM. O contexto do desemvolvimento psicossocial de jogadores de futebol para amputados. Revista Brasileira de Futsal e Futebol. 2017;9(34):221-8. 